\title{
Exploring Molecular Mechanism of Traditional Chinese Medicine Euphorbiae Semen on Reversing of Multidrug Resistance in Leukemia Based on Network Pharmacology Strategy and Molecular Docking Technology
}

\section{Xiao Song}

School of Pharmaceutical Sciences, Shandong University of Traditional Chinese Medicine, Jinan 250355, PR China

Fei Guo

The Affiliated Hospital of Shandong University of Traditional Chinese Medicine, Jinan 250011, PR China

Xiao-Chen Sun

School of Pharmaceutical Sciences, Shandong University of Traditional Chinese Medicine, Jinan 250355, PR China

\section{Shu-Yue Wang}

School of Pharmaceutical Sciences, Shandong University of Traditional Chinese Medicine, Jinan 250355, PR China

\section{Yao-Hui Yuan}

School of Pharmaceutical Sciences, Shandong University of Traditional Chinese Medicine, Jinan 250355, PR China

Chao Zhang ( $\nabla$ tougaotcm@163.com )

School of Pharmaceutical Sciences, Shandong University of Traditional Chinese Medicine, Jinan 250355, PR China https://orcid.org/0000-0001-9533-3737

\section{Hua-Ying Lv}

Shandong College of Traditional Chinese Medicine, Yantai 264199, PR China

\section{Zhi Chen}

School of Pharmaceutical Sciences, Shandong University of Traditional Chinese Medicine, Jinan 250355, PR China

\section{Research}

Keywords: Euphorbiae semen, network pharmacology, molecular docking, leukemia, multidrug resistance

Posted Date: August 11th, 2020

DOI: https://doi.org/10.21203/rs.3.rs-55927/v1

License: (c) (i) This work is licensed under a Creative Commons Attribution 4.0 International License. Read Full License 


\section{Abstract}

Background: Leukemia was listed by the World Health Organization as one of the five most intractable diseases in the world. The multi-drug resistance (MDR) of leukemia cells limits the efficacy of anti-tumor drugs and is the major reason for the chemotherapy failure and recurrence of leukemia chemotherapy. Some studies have shown that Euphorbiae semen (ES) possesses the characteristics of new therapeutic drugs for MDR. However, the molecular mechanisms and active compounds have not yet been fully clarified. Therefore, there is a need for explore its active compounds and demonstrate its mechanisms through network pharmacology and molecular docking technology.

Method: First, the TCMSP database was searched and screened the active compounds of the ES, supplemented with compounds verified by literature, so as to further identify the core compounds in the active ingredient. Simultaneously, the TCMSP and Swiss database were searched to the targets of active compounds, and the targets of reverses leukemia multidrug resistance (RL-MDR) were screened in the relevant databases, such as GeneCards and DrugBank. Then, the targets of active compounds were intersected with RL-MDR targets to obtain potential targets of ES acting on MDR. The compound-target network was constructed by Cytoscape. The target protein-protein interaction network was built using STRING and Cytoscape database. Second, the R language and DAVID database were used to analyse Gene Ontology (GO) biological functions analysis and Kyoto Encyclopedia of Genes and Genomes (KEGG) signal pathways enrichment. Finally, molecular docking method was utilized to investigate the binding activity between the core targets and the active compounds of ES.

Results: Compound-target network mainly contained 22 compounds and 81 corresponding targets. Finally, seven components in ES were selected and 10 core targets were identified; Key targets contained JUN, CASP3, MAOA, AR, PPARG, DRD2, ADRA2A, CHRM2, PTGS2 and MAPK14. G0 enrichment analysis indicated the main biological functions of potential genes of ES in the treatment of MDR. KEGG pathway enrichment analysis showed the main pathways, mainly including apoptosis, pathways in cancer, p53 signaling pathway, VEGF signaling pathway, TNF signaling pathway and PI3K-Akt signaling pathway. Finally, we chose the top 10 common targets for molecular docking with the 7 active compounds of ES. The results of molecular docking indicated that the compounds of ES, which had good affinity with targets.

Conclusion: The molecular mechanism of ES in the treatment of MDR showed the synergistic reaction of multi-compound, multi-target, and multi-pathway of traditional Chinese medicine, which provided ideas for further clinical research.

\section{Introduction}

Leukemia [1] is a kind of malignant clonal disease of hematopoietic stem cells, commonly known as "blood cancer", which ranks first in the incidence of pediatric malignant tumors. The mortality rate ranks first among the malignant tumors that cause death in children and adults under the age of 35, and it is one of the malignant tumors that seriously endanger human health. The majority of therapeutic failures are due to cellular resistance to anti-leukemic drugs. Multidrug resistance (MDR) refers to the phenomenon that tumor cells develop resistance to an anti-tumor drug while producing MDR to anti-tumor drugs that have not been exposed and exposed, different mechanisms of action, and different targets of action [2]. The development of multidrug resistance is one of the main reasons for treatment failure in various cancers [3-5]. Multidrug resistance is a great challenge in cancer treatment, is a detrimental mechanism that induces cancer cells to develop resistance against chemotherapies, which need to be solved [6, 7]. A major mechanism of resistance is the over-expressed in cancer cell plasma membranes of ATP-binding cassette (ABC) transporters, which could efflux pump the chemotherapeutic drugs flow out of the cell [8-10]. Therefore, many anticancer substances cannot effectively accumulate in tumor cells to reach enough therapeutic concentration, resulting in multidrug resistance[11]. Consequently, it is necessary to develop Chinese medicine reversal agents and anti-tumor drugs synergistic reverses leukemia multidrug resistance (RL-MDR). 
Traditional Chinese medicine reversal agent has the characteristics of multi-target, low toxicity and high efficiency, which can be passed through different mechanism reversal of drug resistance, with unlimited research potential. At present, a crucial strategy for solve MDR is to use sensitizer or reversal agents, which are combined with chemotherapeutic drugs [12]. It has become the current focus of researchers. Traditional Chinese herbal, as a complementary and alternative medicine treatment, has been widely applied into cancer treatment. For instance, emodin can reverse the drug resistance of K562 / ADM cells by inhibiting the expression of P - glycoprotein, which suggests that emodin is still potentially effective reversal agents in the treatment of chronic myeloid leukemia resistance [13]. solanine[14]and germacrone[15]were able to MDR. Through inhibiting the expression of $\mathrm{P}$ - glycoprotein and MRP, increasing the concentration of chemotherapeutic drugs in chronic myelogenous leukemia resistant cell lines and enhancing the cytotoxicity of drugs.

Euphorbiae semen also called QianJinzi in Chinese or Euphorbia Lathyris L. in Latin, distributed mainly in North China[16]. It has been widely used for thousands of years for the treatment of warts and tumors[17], antibacterial activity[18], antiviral[19, 20], anti-inflammation activity[21, 22], anti-proliferative, antitumor-promoting and apoptosis inducing activities[23-25], and et al. The main compounds of the ES include Lathyrane-type, diterpenoids, Ingenane-type, diterpenoids, Coumarins, etc. Many studies have been found that Euphorbia diterpenes can be regarded as effective lead compounds for the reversal of MDR [26-28]. It can be seen that ES have certain development value.

Network pharmacology, which is a newly developed strategy first mentioned by Andrew L Hopkins, is a systematic analytical way focuses on searching for relationships of active ingredients and potential targets[29]. Network pharmacology combined with molecular docking technology could be applied in identify active ingredients, predict target, clarify mechanism and pathway of action [30]. Therefore, we explored its possible mechanism of ES in RL-MDR with network pharmacology and molecular docking technology, which provides a reference for further research on the drug treatment of leukemia.

\section{Methods}

\subsection{Screening of compounds from ES}

TCMSP contains 499 herbs registered in the Chinese Pharmacopoeia with 29,384 ingredients, 3,311 targets, and 837 associated diseases and it contains the drug-gene interaction based on both experiment and prediction traditional Chinese Medicine Systems Pharmacology Database and Analysis Platform (TCMSP: https://tcmspw.com/). TCMSP provides lots of information about the Euphorbia semen for us. Therefore, we screened compounds with oral bioavailability $(\mathrm{OB}) \otimes 30 \%$ and Drug-likeness $(\mathrm{DL}) \otimes 0.18$ as bioactive compounds for further study. At the same time, potential compounds which could validate by literatures were replenished.

\subsection{Screening of bioactive compounds potential targets}

The potential targets of ES were predicted on TCMSP and Swiss Target Prediction (http://www.swisstargetprediction.ch/, 2019 version). Only the targets with Probability (Swiss Target Prediction) higher than 0.60 would be selected, the aim of this doing was to ensure the reliability of prediction. The targets of compounds from TCMSP and Swiss were combined and duplicated items were removed. Finally, we obtained the potential targets of ES.

2.3 Screening of potential targets for reverses leukemia multidrug resistance (RL-MDR)

The targets related to RL-MDR were retrieved from the Human Gene Database (Genecards database, https://www.genecards.org/) and DrugBank (DrugBank database, https://www.drugbank.ca/). GeneCards builds a correlation between gene and disease, which provides information about targets related to disease, mutations and polymorphisms, protein-protein interactions, gene expression, pathways and so on[31]. The DrugBank database is a free 
and comprehensive bioinformatics database containing information on detailed drug, drug target, drug action, and drug interaction [32]. The targets obtained from two databases, which were regarded as potential targets of RL-MDR. Ultimately, we use the drawing of Venny2.1 diagram (https://bioinfogp.cnb.csic.es/) to analyze the targets' intersection of compounds and RL-MDR.

\subsection{Construction of Protein-Protein Interaction (PPI) network of targets intersection of RL-MDR and ES}

We constructed a PPI network for the protein targets of ES using the string database (http://www.swisstargetprediction.ch/) with the organism limited to "Homo sapiens". String, a database was used for searching and building the network between proteins and proteins. To collect possible protein-protein interactions (PPI) by uploading 81 common targets that related to RL-MDR and active compounds. The minimum required interaction score is medium confidence (0.400), which was also the default threshold. Then, save the file in TSV format. PPI network of targets intersection of RL-MDR and ES was constructed by using Cytoscape (version 3.7.1, Boston, MA, USA) and the modules of PPI network are clustered by MCODE. Cytoscape is open software, which can graphically display the network and edit it[33]. The Cytoscape software was used to construct a PPI network of targets intersection of active compounds and potential targets. Detailed information was provided in these graphs that compounds and targets were represented by nodes and their interactions were reflected by edges.

\subsection{Gene Ontology (GO) and KEGG pathway enrichment analysis of targets intersection of RL-MDR and ES}

We using the Database for Annotation, Visualization, and Integrated Discovery (David database, https://david.ncifcrf.gov/) to perform GO and KEGG pathway enrichment analyses in this study. GO analysis is the description of genes in different aspects such as the biological process (BP), cellular components (CC), and molecular function (MF). KEGG analysis refers to the analysis of the pathways involved in genes. KEGG pathway was determined have statistically significant and necessary functional mechanisms as $P$ value $\leq 0.05[34$ ]. The ggplot2 was used to visualize the bubble chart, which is a contributed visualization package in the $\mathrm{R}$ programming language.

\subsection{Molecular docking and network analysis}

Molecular docking is a significant pathway that mainly studies intermolecular interactions and predicts their binding mode and affinity[35]. Not only can it be used for drug design in new medicines, but it can also provide keen insights into protein function prediction and other important issues [36]. We screened the top 10 targets related to RL-MDR, downloading protein structure in Uniport (https://www.uniprot.org/) save as PDB formats of proteins. Molecular docking is based on the simulation of ligand-receptor interaction to predict the binding mode and affinity between protein and protein or between small molecule and protein to carry out virtual screening of drug targets and the prediction of pharmacodynamic components. The molecular docking was used to explore the interaction between compounds and the reversal of multidrug resistance in leukemia. To some extent, the mechanism and binding activity of compounds and target proteins were explained. The X-ray crystal structures of key target proteins were searched at the RCSB PDB database (https://www.rcsb.org/) and imported into the "Discovery Studio Client (BIOVIA Co., Ltd, China") software to modify the compound structure. In the "simulation" module of the software, select "apply force field" to add force field for compound. Before docking, the receptor protein should be pretreated, the water molecules should be removed, and then the protein should be prepared. The ligand molecules in the prepared receptor protein were selected, the docking site is centered on the ligand, select the Dock Ligands to dock with the compound, and finish the docking after the running. Screening active compounds with high activity and interaction with key amino acids can illustrate the mechanism and binding activity of compounds with key targets in some degree. The higher the docking score, the better the affinity for protein and ligand. The conformation with the best affinity was selected as the final docking conformation and visualized in Pymol (version 2.3, Chengdu MolDesigner Co., Ltd, China). 


\section{Results}

\subsection{Screening of compounds}

A large number of compounds in single Chinese medicine, we screened 22 by using TCMSP and literature. The detailed information of compounds from literature was provided by TCMSP and PubChem (https://pubchem.ncbi.nlm.nih.gov/). In a word, 22 compounds were screened for further study. The detailed information was shown in Table 1.

\subsection{Target retrieval and analysis}

Based on the above results, we further looked for the targets of these components. A total of 5,201 targets were obtained. 2,565 targets, 1,445 targets were initially screened in DrugBank and GeneCards, respectively. 91 targets of compounds were obtained. 81 targets were obtained after merging and removing duplicate targets, as shown in Figure1 (a). The overlapping 81 targets represented the potential targets for ES treating MDR and the targets were imported into Cytoscape for visualization, as shown in Figure1(b).

\subsection{Construction compound -target Network}

The compound-target network consisted of 102 nodes and 139 interacting edges were established by Cytoscape. Each edge represents the interaction between compound and target and a node with higher degree represents its importance in the network. Beta-Sitosterol, DL-Laudanine, Stigmasterol, Artemetin, Eseramine, ISitosterol, and Euphorbetin were the top 7 bioactive compounds with maximum degree in network, the detailed information of these 7 compounds was shown in Table 2. These targets are considered to be the active targets of ES in the treatment of RL-MDR, as shown in Figure 2. The top 43 targets were screened according to the degree value (Table 3).

\subsection{Construction and analysis of the target PPI network.}

We identified 81 potential targets of ES-related genes of RL-MDR utilize the comparative analysis. A diversified PPI network was created by using the Cytoscape to visualization (Figure 3). This PPI network consisted of 79 nodes and 387 edges. COLQ and PRSS1 were not analyzed in the PPI network, for it does not interact with other proteins. The predicted top 10 with highest degree score were JUN, CASP3, MAOA, AR, PPARG, DRD2, ADRA2A, CHRM2, PTGS2, and MAPK14, which were considered core targets of ES to RL-MDR. Analyze the network by MCODE, six clusters are obtained (Figure. 4). Input these clusters into DAVID for GO enrichment analysis, several RL-MDR related biological processes are returned. The details of the clusters are described in Table4. Take some RL-MDR related biological processes in Cluster A as an example: Genes in Cluster A is related to many biological processes. For example: positive regulation of ERK1and ERK2 cascode, (GO: 0070374) positive regulation of phosphatidylinositol biosynthetic process, (G0: 0010513) positive regulation of release of sequestered calcium ion into cytosol, (GO: 0051281) positive regulation of phosphatidylinositol 3kinase signaling pathway, (GO: 0014068) adenylate cyclase-inhibiting G protein-coupled receptor signaling pathway, (GO: 0007193), et al.

3.5 GO and KEGG pathway enrichment analysis.

The GO and KEGG enrichment analysis were used to comment on the 81 potential targets of ES. We make the level of statistical significance at $P<0.05$. Then, select the top 20 significantly enriched terms in the BP, CC, and MF categories showed in Figure 5. GO enrichment analysis showed that ES can inhibit of adenylate cyclase and a subsequent decrease in the concentration of cyc AMP (cAMP), block the cell signaling pathway to inhibit cell proliferation and promote apoptosis. Additionally, it works on GPCRs, which are closely related to biological behaviors such as the proliferation, invasion, and metastasis of tumors, involving the classical signal pathways such as MAPK, Rap 1 and PI3K/Akt. Additionally, it can promote calcium ion release into the cytosol. It is possible that the role of ES on RL-MDR is through these molecular functions. 
Subsequently, we conducted a KEGG pathway enrichment analysis. The neuroactive ligand-receptor interaction pathway showed the highest number of target connections (count=28), calcium signaling pathway with14 targets, and pathways in cancer with 17 targets, and included p53 signaling pathway, TNF signaling pathway, apoptosis, VEGF signaling pathway, HIF-1 signaling pathway, CAMP signaling pathway, TGF- $\beta$ signaling pathway, Wnt signaling pathway and PI3K/Akt signaling pathway. These pathways play crucial roles in the inhibition of tumor cell growth, differentiation and promote tumor cell apoptosis. Anything else, signaling pathways involved in inflammation and immunity, such as T cell receptor signaling pathway, NF-kB signaling pathway, Jak-STAT signaling pathway and so on. The results demonstrated that the action targets of main bioactive compounds were distributed in different pathways. The "multi-components, multi-targets, and multi-pathways" mutual regulation is the possible mechanism for the reverse leukemia multidrug resistance. Then, the top 20 signaling pathways were visualization in Figure 6. Details are provided in Table 5.

\subsection{Molecular docking verification}

Because 43 potential targets were obtained, the top 10 targets (JUN, CASP3, MAOA, AR, PPARG, DRD2, ADRA2A, CHRM2, PTGS2and MAPK14), which had higher scores, were selected for molecular docking with 7 active ingredients of ES. In this docking assay, apart from ADRA2A with no protein PDB format, nine protein structures were retrieved from Protein Data Bank (PDB), showed in Table 6. The results obtained by the molecular docking software are shown in Table 7. Through molecular docking, it can reveal the interaction between molecules and targets. It provides the most reasonable and effective mass model in structure-activity relationship research. Compounds with the highest docking fraction were selected for plotting, the results shown in Figure 7. It shows that the compounds were tightly bound to the protein residues via various interactions. The results revealed that the nine potential targets can be docked with different compounds respectively, with different docking fraction, which further indicated that ES plays a role through multi-component and multi-target.

\subsection{Construction of compound-target genes-pathway network}

A comprehensive network was constructed with nodes consistent with compounds, targets, pathways, and edges indicating interactions, respectively, in Figures 8 . It can be seen from the figure that an active component not only corresponds to one action target, but also corresponds to one or more active components. The network fully demonstrated that ES possessed multiple components, multiple targets, and multiple pathways against MDR.

\section{Discussion}

Until now, chemical drugs are the main way to treat leukemia, while leukemia cell MDR is an important cause of treatment failure. The mechanism of MDR in tumor cells is a complex process with multiple targets and pathways. Chemical drugs have the deficiency of single target and high toxicity in reversing MDR of tumor cells. Traditional Chinese medicine (TCM) can reverse MDR of leukemia cells due to its advantages of multi-pathway and low toxicity, which provides another way to seek for efficient reversing agents. TCM has its unique advantages to treat complex disease. Nevertheless, multicompound and multi-target characteristics of TCM also brought a lot of difficulties for Chinese medicine research and restrained the development of TCM. By changing from "one-target, one-drug" analysis to "network-targeted, multicomponent" analysis, network pharmacology is a powerful way for the molecular mechanism of TCM [37]. Therefore, the development of network pharmacology, which can predict the interaction of multiple drug targets, may be the key to the success of drug discovery against complex diseases in the future. In our study, molecular docking verification was added to the network pharmacology as complement to predict the herb targets.

According to compound-target network, beta-Sitosterol, DL-Laudanine, Stigmasterol, Artemetin, Eseramine, Sitosterol and Euphorbetin were compounds with top seven degree among the network. Beta-sitosterol, a phytosterol induces anticancer properties in different cancers based on different mechanisms [38]. It is indicated that beta-sitosterol induced G0/G1 cell 
cycle arrest in NSCLC cells possibly by inactivating the TGF- $\beta /$ Smad2/3/c-Myc pathway [39]. Beta-Sitosterol which has been shown to have reverse MDR on leukemia via induces $\mathrm{G} 2 / \mathrm{M}$ arrest, endoreduplication, and apoptosis through the Bcl2 and PI3K/Akt signaling pathways. It could be considered as good candidate for the development of novel P-gp/MDR1 reversal agents which may enhance the accumulation and efficacy of chemotherapy agents. It is reported that artemetin is a potential radiosensitizer which inhibits proliferation by inducing apoptosis in human myeloid leukemia cells[40], and inhibits cell cycle progression at G2/M phase and induces apoptosis in mammalian cancer cells[41]. These reports indicated that the compounds we have screened can indeed play a role in different ways.

Our results predict that compounds exert therapeutic effects against MDR, at least in part, by modulating the function of the following proteins: JUN, CASP3, MAOA, AR, PPARG, DRD2, ADRA2A, CHRM2, PTGS2, MAPK14. Functions of intersect targets can be classified into steroid hormone receptor activation and regulate tumor cell growth and apoptosis and drug resistance. For example, as an important member of AP-1 transcription factor family, JUN is involved in growth, metastasis, and drug resistance of cancer[42]. PTGS2 is also known as cyclooxygenase-2 (COX-2), and Cox-2 is a ratelimiting enzyme that regulates prostaglandin synthesis during arachidonic acid metabolism and is mainly involved in various inflammatory reactions of the body [43]. In addition, PTGS2 can activate nuclear factor kappa B (NF - KB) signaling pathway and reduce DNA damage after radiotherapy [44]. MAPK is a class of serine/threonine protein kinases, which is an important intracellular signal transduction system, mediating various processes of cell differentiation, proliferation, division and apoptosis in the body, and is related to the occurrence, development and metastasis of various tumors [45]. Caspase-3 controls AML1-ETO-driven leukemogenesis via autophagy modulation in a ULK1-dependent manner [46]. These studies indicate that biological functions of the targets play important roles in RL-MDR.

Furthermore, we performed functional enrichment analyses, including GO and KEGG pathway enrichment analysis, to clarify the multiple mechanisms of ES reverse MDR. The pathways involved in the KEGG enrichment pathway were mainly the pathways in cancer, apoptosis, p53 signaling pathway, T cell receptor signaling pathway, TNF signaling pathway, VEGF signaling pathway and PI3K/Akt signaling pathway. TNF signaling pathway was an important pathway in inflammatory response [47], in which related factor receptors can also induce apoptosis. T cell receptor (TCR) activation can promote many signal transduction cascades and ultimately determine cell fate by regulating cytokine production, cell survival, proliferation, and differentiation[48]. The PI3K/Akt signaling pathway was a key pathway in our study. Studies have found that the PI3K/Akt signaling pathway is continuously activated in leukemia cells, and the abnormal activation of PI3K/Akt signaling pathway is closely related to multidrug resistance in leukemia. Inhibition of activation of this pathway can inhibit proliferation of tumor cells and directly regulate the apoptosis process of leukemia cells [49]. To sum up, it is speculated that ES might exert RL-MDR effect by acting on the related signaling pathways.

Molecular docking is the most widely used method for calculating protein-ligand interactions. Through network pharmacology combined with molecular docking technology, the possible active components and molecular mechanisms of ES for reversing MDR were systematically screened in this study to provide a new breakthrough point for the treatment of MDR. More and more evidences have shown that ES may have significant potential to RL-MDR by a combination of multi-components, multi-targets, and multi-pathways. Nevertheless, its rationality is only preliminarily explained in this study that still has some limitations.

\section{Conclusion}

In clinical application, the complexity of herbal compounds, and they act on a variety of complex targets and pathways, which makes clinical application and research on the active ingredients of Chinese traditional medicines difficult. Network pharmacology and molecular docking method, although they have many problems that need to be solved, which provide new ideas and new methods for thinking of research on the material basis and mechanisms of traditional Chinese medicines[50]. This research explored the mechanism of the effects of ES reverse MDR based on these methods for the first time. The results indicated that the ES could play pharmacological roles in reverse leukemia multidrug resistance 
through multiple targets, multiple channels, and multiple pathways, including apoptosis, pathways in cancer, TNF, inflammation, and immune stimulation. The docking results further demonstrated that main compounds displayed good affinity to the critical targets. Furthermore, this study not only provides a solid foundation for clinical application, but also provides a reference for further research on the drug treatment of leukemia. However, there was also shortcoming in this study, which was failed to carry out further clinical trials and verification studies. Therefore, our future research will pay attention to in vivo, in vitro experiments and clinical trials to verify these results.

\section{Declarations}

Acknowledgments: Not applicable.

Funding: This work was supported by Key Research and Development Plan of Shandong Province (No. 2019GSF108171), National Natural Science Foundation of China (No. 81603298) and A Project of Shandong Province Traditional Chinese Medicine Science and Technology Development Program (No. 2015-218 and 2019-028).

Author Contribution: XS and FG designed and conducted the study with equal contribution. CZ and ZC supervised the study. XCS, SYW, YHY and HYL provided the technical support and advices for the study. XS wrote the paper. All authors read and approval of the final manuscript.

Availability of data and materials: The data used to support the results of this study can be obtained from the first author on reasonable request.

Ethics approval and consent to participate: Not applicable.

Conflicts of Interest: The authors declare no conflict of interest.

Consent for publication: Not applicable.

\section{References}

1. Greaves M. Leukaemia 'firsts' in cancer research and treatment. Nat Rev Cancer. 2016;16(3):163-72.

2. Kunjachan S, Rychlik B, Storm G, Kiessling F, Lammers T. Multidrug resistance: Physiological principles and nanomedical solutions. Adv Drug Deliv Rev. 2013;65(13-14):1852-65.

3. Kartal-Yandim M, Adan-Gokbulut A, Baran Y. Molecular mechanisms of drug resistance and its reversal in cancer. Crit Rev Biotechnol. 2016;36(4):716-26.

4. Robak P, Drozdz I, Szemraj J, Robak T. Drug resistance in multiple myeloma. Cancer Treat Rev. 2018;70:199-208.

5. Hasanovic A, Mus-Veteau I. Targeting the Multidrug Transporter Ptch1 Potentiates Chemotherapy Efficiency. Cells. 2018;7(8).

6. Szakacs G, Paterson JK, Ludwig JA, Booth-Genthe C, Gottesman MM. Targeting multidrug resistance in cancer. Nat Rev Drug Discov. 2006;5(3):219-34.

7. Housman G, Byler S, Heerboth S, Lapinska K, Longacre M, Snyder N, et al. Drug resistance in cancer: an overview. Cancers (Basel). 2014;6(3):1769-92.

8. Kosztyu P, Dolezel P, Mlejnek P. Can P-glycoprotein mediate resistance to nilotinib in human leukaemia cells? Pharmacol Res. 2013;67(1):79-83. 
9. Rebucci M, Michiels C. Molecular aspects of cancer cell resistance to chemotherapy. Biochem Pharmacol. 2013;85(9):1219-26.

10. Gottesman MM, Ling V. The molecular basis of multidrug resistance in cancer: the early years of P-glycoprotein research. FEBS Lett. 2006;580(4):998-1009.

11. Feng Y, Hu Y, Cen J, Darshika KN, Fang W, Li Y, et al. HZO8 inhibits the multi-drug resistance on multiple sites as the substrate of p-glycoprotein. Eur J Pharmacol. 2013;712(1-3):53-9.

12. Suttana W, Mankhetkorn S, Poompimon W, Palagani A, Zhokhov S, Gerlo S, et al. Differential chemosensitization of Pglycoprotein overexpressing K562/Adr cells by withaferin A and Siamois polyphenols. Mol Cancer. 2010;9:99.

13. Min H, Niu M, Zhang W, Yan J, Li J, Tan X, et al. Emodin reverses leukemia multidrug resistance by competitive inhibition and downregulation of P-glycoprotein. PLoS One. 2017;12(11):e0187971.

14. Yi YJ, Jia XH, Zhu C, Wang JY, Chen JR, Wang H, et al. Solanine reverses multidrug resistance in human myelogenous leukemia K562/ADM cells by downregulating MRP1 expression. Oncol Lett. 2018;15(6):10070-6.

15. Pan J, Miao D, Chen L. Germacrone reverses adriamycin resistance in human chronic myelogenous leukemia K562/ADM cells by suppressing MDR1 gene/P-glycoprotein expression. Chemico-Biological Interactions. 2018;288:32-7.

16. Tian-Xi Zhou a-HB, aQin-Gao Ma,aGuo-Wei Qin,a,* Chu-Tao Che,bYang Lv,c, Zhengc CWQ-T. Langduin C, a novel dimeric diterpenoid from the roots of Euphorbia fischeriana. TET LETTERS.44(12):135-7.

17. J. H. Plants used against cancer. A survey. Lloydia.8(9):1353-8.

18. Yang L, Ding W, Xu Y, Wu D, Li S, Chen J, et al. New Insights into the Antibacterial Activity of Hydroxycoumarins against Ralstonia solanacearum. Molecules. 2016;21(4):468.

19. Cottiglia F, Loy G, Garau D, Floris C, Casu M, Pompei R, et al. Antimicrobial evaluation of coumarins and flavonoids from the stems of Daphne gnidium L. Phytomedicine. 2001;8(4):302-5.

20. Cheng HY, Lin TC, Yang CM, Wang KC, Lin LT, Lin CC. Putranjivain A from Euphorbia jolkini inhibits both virus entry and late stage replication of herpes simplex virus type 2 in vitro. J Antimicrob Chemother. 2004;53(4):577-83.

21. Liu Z, Liu J, Zhao K, Shi Q, Zuo T, Wang G, et al. Role of Daphnetin in Rat Severe Acute Pancreatitis Through the Regulation of TLR4/NF-[Formula: see text]B Signaling Pathway Activation. Am J Chin Med. 2016;44(1):149-63.

22. Liu ZY, Liu J, Zhao KL, Wang LK, Shi Q, Zuo T, et al. Protective effects of daphnetin on sodium taurocholateinduced severe acute pancreatitis in rats. Mol Med Rep. 2014;9(5):1709-14.

23. ROZÁLIA PUSZTAI1 M-JUF, NOÉLIA DUARTE2, HELGA ENGI1and JOSEPH MOLNAR1. Macrocyclic Lathyrane Diterpenes as Antitumor Promoters. ANTICANCER RESEARCH. 2007;27:201-6.

24. Miyata S, Wang LY, Yoshida C, Kitanaka S. Inhibition of cellular proliferation by diterpenes, topoisomerase II inhibitor. Bioorg Med Chem. 2006;14(6):2048-51.

25. Kuo PL, Cho CY, Hsu YL, Lin TC, Lin CC. Putranjivain A from Euphorbia jolkini inhibits proliferation of human breast adenocarcinoma MCF-7 cells via blocking cell cycle progression and inducing apoptosis. Toxicol Appl Pharmacol. 2006;213(1):37-45. 
26. OLGA WESOLOWSKA1 JW, NOÉLIA DUARTE2, MARIA-JOSÉ U. FERREIRA2and KRYSTYNA MICHALAK1. Inhibition of MRP1 Transport Activity by Phenolic and Terpenic Compounds Isolated from Euphorbia species. ANTICANCER RESEARCH. 2007;27: 4127-34.

27. Zhang JY, Mi YJ, Chen SP, Wang F, Liang YJ, Zheng LS, et al. Euphorbia factor L1 reverses ABCB1-mediated multidrug resistance involving interaction with ABCB1 independent of ABCB1 downregualtion. J Cell Biochem. 2011;112(4):1076-83.

28. Giovanni Appendino CDP, Gwenae,Ile Conseil,Olov Sterner,Enrico,Mercalli,Charles Dumontetand Attilio Di Pietro. A New P-Glycoprotein Inhibitor from the Caper Spurge (Euphorbia lathyris). J Nat Prod 2003;66(6):140-2.

29. Hopkins AL. Network pharmacology. NATURE BIOTECHNOLOGY. 2007;25(10):1110-1.

30. Azike CG, Charpentier PA, Lui EM. Stimulation and suppression of innate immune function by American ginseng polysaccharides: biological relevance and identification of bioactives. Pharm Res. 2015;32(3):876-97.

31. Fishilevich S, Zimmerman S, Kohn A, Iny Stein T, Olender T, Kolker E, et al. Genic insights from integrated human proteomics in GeneCards. Database (Oxford). 2016;2016.

32. Wishart DS, Feunang YD, Guo AC, Lo EJ, Marcu A, Grant JR, et al. DrugBank 5.0: a major update to the DrugBank database for 2018. Nucleic Acids Res. 2018;46(D1):D1074-D82.

33. Su G, Morris JH, Demchak B, Bader GD. Biological network exploration with Cytoscape 3. Curr Protoc Bioinformatics. 2014;47:8 13 1-24.

34. Liang B, Li C, Zhao J. Identification of key pathways and genes in colorectal cancer using bioinformatics analysis. Med Oncol. 2016;33(10):111.

35. NOURELDEIN MH. In silico discovery of a perilipin 1 inhibitor to be used as a new treatment for obesity. Eur Rev Med Pharmacol Sci. 2014;18:457-60.

36. Pires DE, Ascher DB. CSM-lig: a web server for assessing and comparing protein-small molecule affinities. Nucleic Acids Res. 2016;44(W1):W557-61.

37. Fang HY, Zeng HW, Lin LM, Chen X, Shen XN, Fu P, et al. A network-based method for mechanistic investigation of Shexiang Baoxin Pill's treatment of cardiovascular diseases. Sci Rep. 2017;7:43632.

38. Bin Sayeed MS, Ameen SS. Beta-Sitosterol: A Promising but Orphan Nutraceutical to Fight Against Cancer. Nutr Cancer. 2015;67(8):1214-20.

39. Wang X, Li M, Hu M, Wei P, Zhu W. BAMBI overexpression together with beta-sitosterol ameliorates NSCLC via inhibiting autophagy and inactivating TGF-beta/Smad2/3 pathway. Oncol Rep. 2017;37(5):3046-54.

40. W. G. KO THK, S. J. LEE, N. Y. KIM, Y. C. KIM, D. H. SOHN and B. H. LEE *. Polymethoxyflavonoids from Vitex rotundifolia inhibit proliferation by inducing apoptosis in human myeloid leukemia cells. Food and Chemical Toxicology. 2000;38(3):861-5.

41. Li WX, Cui CB, Cai B, Wang HY, Yao XS. Flavonoids from Vitex trifolia L. inhibit cell cycle progression at G2/M phase and induce apoptosis in mammalian cancer cells. J Asian Nat Prod Res. 2005;7(4):615-26.

42. Meng S, Wang G, Lu Y, Fan Z. Functional cooperation between HIF-1alpha and c-Jun in mediating primary and acquired resistance to gefitinib in NSCLC cells with activating mutation of EGFR. Lung Cancer. 2018;121:82-90. 
43. Cui Q, Zhang YL, Ma YH, Yu HY, Zhao XZ, Zhang LH, et al. A network pharmacology approach to investigate the mechanism of Shuxuening injection in the treatment of ischemic stroke. J Ethnopharmacol. 2020;257:112891.

44. Tan C, Liu L, Liu X, Qi L, Wang W, Zhao G, et al. Activation of PTGS2/NF-kappaB signaling pathway enhances radiation resistance of glioma. Cancer Med. 2019;8(3):1175-85.

45. Gangadhara S, Smith C, Barrett-Lee P, Hiscox S. 3D culture of Her2+ breast cancer cells promotes AKT to MAPK switching and a loss of therapeutic response. BMC Cancer. 2016;16:345.

46. Man N, Tan Y, Sun XJ, Liu F, Cheng G, Greenblatt SM, et al. Caspase-3 controls AML1-ETO-driven leukemogenesis via autophagy modulation in a ULK1-dependent manner. Blood. 2017;129(20):2782-92.

47. Kashyap D, Mittal S, Sak K, Singhal P, Tuli HS. Molecular mechanisms of action of quercetin in cancer: recent advances. Tumour Biol. 2016;37(10):12927-39.

48. Burbach BJ, Medeiros RB, Mueller KL, Shimizu Yo. T-cell receptor signaling to integrins. Immunological Rev. 2007;218:65-81.

49. Arrigoni E, Del Re M, Galimberti S, Restante G, Rofi E, Crucitta S, et al. Concise Review: Chronic Myeloid Leukemia: Stem Cell Niche and Response to Pharmacologic Treatment. Stem Cells Transl Med. 2018;7(3):305-14.

50. Fleischer T, Chang TT, Chiang JH, Chang CM, Hsieh CY, Yen HR. Adjunctive Chinese Herbal Medicine therapy improves survival of patients with chronic myeloid leukemia: a nationwide population-based cohort study. Cancer Med.

2016;5(4):640-8.

\section{Tables}




\begin{tabular}{|c|c|c|c|c|c|}
\hline Number & $\begin{array}{l}\text { Molecule ID/ } \\
\text { PubChem CID }\end{array}$ & Molecule name & Molecule weight & $\mathrm{OB}(\%)$ & $\mathrm{DL}$ \\
\hline E1 & MOL002587 & Euphorbetin & 354.28 & 35.89 & 0.54 \\
\hline E2 & MOL002597 & Euphol & 426.80 & 42.12 & 0.75 \\
\hline E3 & MOL000358 & beta-Sitosterol & 414.79 & 36.91 & 0.75 \\
\hline E4 & MOL000359 & Sitosterol & 414.79 & 36.91 & 0.75 \\
\hline E5 & MOL000449 & Stigmasterol & 412.77 & 43.83 & 0.76 \\
\hline E6 & MOL005220 & Eseramine & 318.42 & 45.89 & 0.31 \\
\hline E7 & MOL005221 & Euphorbia factor Ti2 & 538.74 & 35.18 & 0.82 \\
\hline E8 & MOL005223 & DL-Laudanine & 343.46 & 69.09 & 0.37 \\
\hline E9 & MOL005228 & Euphorbia steroid & 552.72 & 34.74 & 0.83 \\
\hline E10 & MOL005229 & Artemetin & 388.40 & 49.55 & 0.48 \\
\hline E11 & MOL005230 & lathyrol & 334.50 & 50.76 & 0.34 \\
\hline E12 & MOL005231 & Isoeuphorbetin & 354.28 & 39.71 & 0.54 \\
\hline E13 & 101306826 & Euphorbia Factor L1 & 552.7 & - & - \\
\hline E14 & 101071473 & Euphorbia Factor L2 & 642.7 & - & - \\
\hline E15 & 10577938 & Euphorbia Factor L3 & 522.6 & - & - \\
\hline E16 & 124511085 & Euphorbia Factor L4 & 586.8 & - & - \\
\hline E17 & 56841025 & Euphorbia Factor L5 & 586.8 & - & - \\
\hline E18 & 6442562 & Euphorbia Factor L6 & 548.7 & - & - \\
\hline E19 & 74962706 & Euphorbia Factor L7a & 548.7 & - & - \\
\hline E20 & 74962707 & Euphorbia Factor L7b & 580.7 & - & - \\
\hline E21 & 85236453 & Euphorbia Factor L8 & 523.6 & - & - \\
\hline E22 & 102004672 & Euphorbia Factor L9 & 643.7 & - & _ \\
\hline
\end{tabular}




\begin{tabular}{|lllll|}
\hline \multicolumn{4}{|c|}{ Table 2: Topological parameter of core active compounds in compound-target network } \\
\hline Number & compounds & Degree & Betweenness & Closeness \\
\hline E3 & MOL000358 & 34 & 0.40 & 0.47 \\
\hline E8 & MOL005223 & 30 & 0.32 & 0.42 \\
\hline E5 & MOL000449 & 25 & 0.26 & 0.42 \\
\hline E10 & MOL005229 & 20 & 0.26 & 0.40 \\
\hline E6 & MOL005220 & 14 & 0.15 & 0.36 \\
\hline E4 & MOL000359 & 6 & 0.09 & 0.31 \\
\hline E1 & MOL002587 & 3 & 0.02 & 0.33 \\
\hline
\end{tabular}




\begin{tabular}{|c|c|c|}
\hline Gene names & Degree & Protein names \\
\hline JUN & 24 & Transcription factor AP-1 \\
\hline CASP3 & 21 & Caspase-3 \\
\hline MAOA & 18 & Amine oxidase [flavin-containing] A \\
\hline AR & 17 & Androgen Receptor \\
\hline PPARG & 17 & Peroxisome proliferator activated receptor gamma \\
\hline DRD2 & 17 & $\mathrm{D}(2)$ dopamine receptor \\
\hline ADRA2A & 17 & Alpha-2A adrenergic receptor \\
\hline CHRM2 & 17 & Muscarinic acetylcholine receptor M2 \\
\hline PTGS2 & 17 & Prostaglandin G/H synthase 2 \\
\hline MAPK14 & 16 & Mitogen-activated protein kinase 14 \\
\hline OPRM1 & 16 & Mu-type opioid receptor \\
\hline HTR2C & 16 & 5-hydroxytryptamine $2 \mathrm{C}$ receptor \\
\hline SLC6A2 & 16 & Sodium-dependent noradrenaline transporter \\
\hline SLC6A3 & 15 & Sodium-dependent dopamine transporter \\
\hline ADRA2C & 15 & Alpha-2C adrenergic receptor \\
\hline HTR1B & 15 & 5-hydroxytryptamine 1B receptor \\
\hline MAOB & 14 & Amine oxidase [flavin-containing] B \\
\hline ADRA1B & 14 & Alpha-1B adrenergic receptor \\
\hline F2 & 14 & Thrombin \\
\hline DRD3 & 14 & $\mathrm{D}(3)$ dopamine receptor \\
\hline HTR2A & 14 & 5-hydroxytryptamine $2 \mathrm{~A}$ receptor \\
\hline CHRM1 & 14 & Muscarinic acetylcholine receptor M1 \\
\hline GPER1 & 14 & Estrogen receptor \\
\hline PGR & 13 & Progesterone receptor \\
\hline OPRD1 & 13 & Delta-type opioid receptor \\
\hline ADRA1A & 13 & Alpha-1 $\mathrm{A}$ adrenergic receptor \\
\hline ADRA1D & 13 & Alpha-1D adrenergic receptor \\
\hline ADRA2B & 13 & Alpha-2B adrenergic receptor \\
\hline NCOA1 & 12 & Nuclear receptor coactivator 1 \\
\hline CDK2 & 12 & Sodium channel protein type 5 subunit alpha \\
\hline PRKACA & 12 & mRNA of PKA Catalytic Subunit C-alpha \\
\hline CHRM4 & 12 & Muscarinic acetylcholine receptor M4 \\
\hline
\end{tabular}

Page 14/23 


\begin{tabular}{|lll|}
\hline NCOA2 & 11 & Nuclear receptor coactivator 2 \\
\hline ESR2 & 11 & Mu-type opioid receptor \\
\hline GSK3B & 11 & Prostaglandin G/H synthase 2 \\
\hline HSP90AB1 & 11 & Heat shock protein HSP 90 \\
\hline ADRB2 & 11 & Beta-2 adrenergic receptor \\
\hline MAP2 & 11 & Microtubule-associated protein 2 \\
\hline CASP8 & 10 & Caspase-8 \\
\hline CASP9 & 10 & Caspase-9 \\
\hline NOS2 & 10 & Nitric oxide synthase, inducible \\
\hline CHRNA7 & 10 & Neuronal acetylcholine receptor protein, alpha-7 chain \\
\hline CHRM5 & 10 & Muscarinic acetylcholine receptor M5 \\
\hline
\end{tabular}

\begin{tabular}{|c|c|c|c|c|}
\hline \multirow[t]{2}{*}{ Cluster } & Targets contained in class clusters & $\begin{array}{l}\text { MCODE } \\
\text { Score }\end{array}$ & $\begin{array}{l}\text { Class } \\
\text { cluster } \\
\text { contains }\end{array}$ & $\begin{array}{l}\text { Class } \\
\text { cluster } \\
\text { contains }\end{array}$ \\
\hline & & & $\begin{array}{l}\text { Number } \\
\text { of } \\
\text { nodes }\end{array}$ & $\begin{array}{l}\text { Number } \\
\text { of edges }\end{array}$ \\
\hline a & 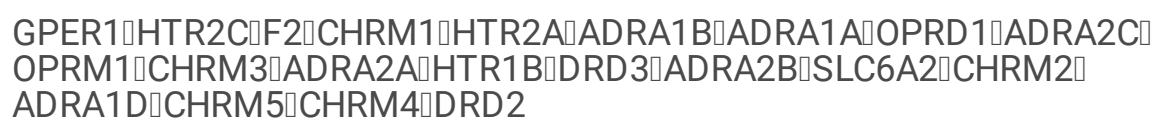 & 10.400 & 21 & 104 \\
\hline b & 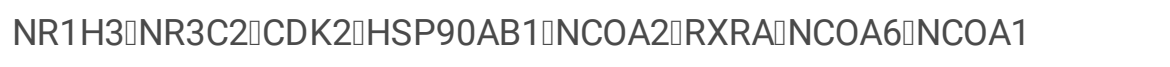 & 4.286 & 8 & 15 \\
\hline c & NOS2ロPPARG!TGFB1"MAPK14 & 4.000 & 4 & 6 \\
\hline d & BCL2ロCASP3ロCASP9ロBAX & 4.000 & 4 & 6 \\
\hline e & GSK3B $\square A B C B 1 \square T O P 2 A \square A R \square J U N$ & 3.500 & 5 & 7 \\
\hline$f$ & SCN5AワKCNMA1ロKCNH2 & 3.000 & 3 & 3 \\
\hline
\end{tabular}


Table 5: Enrichment analysis of KEGG signaling pathway associated with the targets of ES

\begin{tabular}{llll}
\hline Term & Count & $\begin{array}{l}\text { P- } \\
\text { value }\end{array}$ & Group genes \\
$\begin{array}{l}\text { hsa04080:Neuroactive } \\
\text { ligand-receptor } \\
\text { interaction }\end{array}$ & 28 & $\begin{array}{l}8.22 \mathrm{E}- \\
\text { 20 }\end{array}$ & $\begin{array}{l}\text { OPRM1,DRD3,DRD2,PRSS1,HTR1B,ADRA2A, } \\
\text { CHRNA7,ADRA2C,ADRA2B,CHRNA2,GABRA2, }\end{array}$ \\
& & $\begin{array}{l}\text { GABRA1,GABRA3,GABRA5, ADRB2, CHRM5, ADRB1, } \\
\text { CHRM4,CHRM3,CHRM2,CHRM1,F2,ADRA1B, } \\
\text { ADRA1A,HTR2C,ADRA1D,HTR2A,OPRD1 }\end{array}$
\end{tabular}

hsa04020:Calcium

signaling pathway

hsa05200:Pathways in $\quad 17$

cancer

hsa05210:Colorectal

cancer

hsa04022:cGMP-PKG

signaling pathway

hsa04725:Cholinergic

synapse

hsa04726:Serotonergic

synapse

hsa04970:Salivary

secretion

hsa04261:Adrenergic

signaling in

cardiomyocytes

hsa05161:Hepatitis B

hsa05030:Cocaine

addiction

hsa04932:Non-alcoholic 9

fatty liver disease

(NAFLD)

hsa04024:cAMP

signaling pathway

hsa05222:Small cell lung 7 cancer

hsa04923:Regulation of 6

lipolysis in adipocytes

hsa05215:Prostate

cancer

hsa05032:Morphine

addiction

hsa04728:Dopaminergic 8

synapse

hsa04210:Apoptosis

hsa04915:Estrogen
10

14 3.58E-

08

$17 \quad 2.48 \mathrm{E}-$

06

8

$3.49 \mathrm{E}-$ 06

$11 \quad 5.51 \mathrm{E}-$ 06

$9 \quad 2.01 \mathrm{E}-$

05

$92.01 \mathrm{E}-$

05

8 3.12E-

05

$99.59 \mathrm{E}-$

05

9

6

04

$9 \quad 1.80$

04

$1.36 \mathrm{E}$ 04

$1.55 \mathrm{E}$

04

2.23E-

04

2.62E-

04

\section{$2.94 \mathrm{E}-$}

04

7

$3.16 \mathrm{E}-$

04

7

$3.79 \mathrm{E}$

04

8

$3.85 \mathrm{E}-$

04

6

4.74E-

04

7 5.97E-
CHRM5, ADRB2, ADRB1, CHRM3, CHRM2, CHRM1, ADRA1B, ADRA1A, CHRNA7, PRKACA, NOS2, HTR2C, ADRA1D, HTR2A

HSP90AB1, PIK3CG, AR, PTGS2, RXRA, PPARG, CDK2, TGFB1, CASP3, CASP9, JUN, BAX, GSK3B, BCL2, CASP8, PRKACA, NOS2

PIK3CG, CASP3, CASP9, JUN, GSK3B, BAX, BCL2, TGFB1

KCNMA1, ADRB2, ADRB1, ADRA2A, ADRA1B, ADRA1A, PDE3A, ADRA2C, ADRA2B, ADRA1D, OPRD1

PIK3CG, CHRM5, CHRM4, CHRM3, CHRM2, CHRM1, BCL2, PRKACA, CHRNA7

HTR1B, CASP3, PTGS2, MAOA, PTGS1, MAOB, PRKACA, HTR2C, HTR2A

KCNMA1, ADRB2, ADRB1, CHRM3, ADRA1B, ADRA1A, PRKACA, ADRA1D

ADRB2, ADRB1, MAPK14, BCL2, ADRA1B, ADRA1A, PRKACA, SCN5A, ADRA1D

PIK3CG, CASP3, CASP9, JUN, BAX, BCL2, CASP8, TGFB1, CDK2

DRD2, SLC6A3, JUN, MAOA, MAOB, PRKACA

PIK3CG, CASP3, JUN, RXRA, GSK3B, BAX, CASP8, TGFB1, NR1H3

PIK3CG, HTR1B, ADRB2, ADRB1, DRD2, CHRM2, JUN, CHRM1, PRKACA, PDE3A

PIK3CG, CASP9, PTGS2, RXRA, BCL2, NOS2, CDK2

PIK3CG, ADRB2, ADRB1, PTGS2, PTGS1, PRKACA

PIK3CG, HSP90AB1, AR, CASP9, GSK3B, BCL2, CDK2

OPRM1, GABRA2, GABRA1, GABRA3, GABRA5, PRKACA, PDE3A

DRD3, DRD2, MAPK14, SLC6A3, GSK3B, MAOA, MAOB, PRKACA

PIK3CG, CASP3, CASP9, BAX, BCL2, CASP8

PIK3CG, CASP3, CASP9, BAX, BCL2, CASP8

PIK3CG, OPRM1, HSP90AB1, JUN, PRKACA, ESR2, GPER1

Page 16/23 


\begin{tabular}{|c|c|c|c|}
\hline \multicolumn{2}{|l|}{ signaling pathway } & \multicolumn{2}{|l|}{04} \\
\hline $\begin{array}{l}\text { hsa04723:Retrograde } \\
\text { endocannabinoid } \\
\text { signaling }\end{array}$ & 7 & $\begin{array}{l}6.64 \mathrm{E}- \\
04\end{array}$ & GABRA2, GABRA1, PTGS2, GABRA3, MAPK14, GABRA5, PRKACA \\
\hline $\begin{array}{l}\text { hsa04115:p53 signaling } \\
\text { pathway }\end{array}$ & 6 & $\begin{array}{l}6.79 \mathrm{E}- \\
04\end{array}$ & CASP3, CASP9, BAX, CASP8, CHEK1, CDK2 \\
\hline $\begin{array}{l}\text { hsa05033:Nicotine } \\
\text { addiction }\end{array}$ & 5 & $\begin{array}{l}7.99 \mathrm{E}- \\
04\end{array}$ & GABRA2, GABRA1, GABRA3, GABRA5, CHRNA7 \\
\hline hsa05145:Toxoplasmosis & 7 & 0.0010 & CASP3, CASP9, MAPK14, BCL2, CASP8, NOS2, TGFB1 \\
\hline $\begin{array}{l}\text { hsa04919:Thyroid } \\
\text { hormone signaling } \\
\text { pathway }\end{array}$ & 7 & 0.0013 & PIK3CG, NCOA1, NCOA2, CASP9, RXRA, GSK3B, PRKACA \\
\hline $\begin{array}{l}\text { hsa05014:Amyotrophic } \\
\text { lateral sclerosis (ALS) }\end{array}$ & 5 & 0.0019 & CASP3, CASP9, MAPK14, BAX, BCL2 \\
\hline $\begin{array}{l}\text { hsa04914:Progesterone- } \\
\text { mediated oocyte } \\
\text { maturation }\end{array}$ & 6 & 0.0022 & PIK3CG, HSP90AB1, PGR, MAPK14, PRKACA, CDK2 \\
\hline hsa05152:Tuberculosis & 8 & 0.0026 & CASP3, CASP9, MAPK14, BAX, BCL2, CASP8, NOS2, TGFB1 \\
\hline hsa04924:Renin secretion & 5 & 0.0046 & KCNMA1, ADRB2, ADRB1, PRKACA, PDE3A \\
\hline $\begin{array}{l}\text { hsa05142:Chagas } \\
\text { disease (American } \\
\text { trypanosomiasis) }\end{array}$ & 6 & 0.0048 & PIK3CG, MAPK14, JUN, CASP8, NOS2, TGFB1 \\
\hline $\begin{array}{l}\text { hsa05031:Amphetamine } \\
\text { addiction }\end{array}$ & 5 & 0.0051 & SLC6A3, JUN, MAOA, MAOB, PRKACA \\
\hline $\begin{array}{l}\text { hsa04668:TNF signaling } \\
\text { pathway }\end{array}$ & 6 & 0.0054 & PIK3CG, CASP3, PTGS2, MAPK14, JUN, CASP8 \\
\hline $\begin{array}{l}\text { hsa05203:Viral } \\
\text { carcinogenesis }\end{array}$ & 8 & 0.0058 & PIK3CG, CASP3, JUN, BAX, CASP8, PRKACA, CHEK1, CDK2 \\
\hline hsa04976:Bile secretion & 5 & 0.0060 & HMGCR, RXRA, PRKACA, ABCB1, CA2 \\
\hline hsa05140:Leishmaniasis & 5 & 0.0066 & PTGS2, MAPK14, JUN, NOS2, TGFB1 \\
\hline $\begin{array}{l}\text { hsa04722:Neurotrophin } \\
\text { signaling pathway }\end{array}$ & 6 & 0.0087 & PIK3CG, MAPK14, JUN, GSK3B, BAX, BCL2 \\
\hline
\end{tabular}




\begin{tabular}{|lll|}
\hline \multicolumn{3}{|l|}{ Table 6: Information from UniProt of targets' intersection of ES and RL-MDR } \\
\hline Number & Target & Uniport \\
\hline 1 & JUN & $2 \mathrm{G01}$ \\
\hline 2 & CASP3 & $1 \mathrm{NME}$ \\
\hline 3 & MAOA & $2 \mathrm{ZYY}$ \\
\hline 4 & AR & $1 \mathrm{E} 3 \mathrm{G}$ \\
\hline 5 & PPARG & $6 \mathrm{IZN}$ \\
\hline 6 & DRD2 & $6 \mathrm{CM} 4$ \\
\hline 7 & ADRA2A & \\
\hline 8 & CHRM2 & $5 \mathrm{KKC}$ \\
\hline 9 & PTGS2 & $5 \mathrm{IKR}$ \\
\hline 10 & MAPK14 & $2 \mathrm{Y} 1 \mathrm{X}$ \\
\hline
\end{tabular}

\begin{tabular}{|c|c|c|c|c|c|c|c|c|c|}
\hline Compound & AR & CASP3 & JUN & MAPK14 & MAOA & PTGS2 & CHRM2 & DRD2 & PPARG \\
\hline $\begin{array}{l}\text { beta- } \\
\text { Sitosterol }\end{array}$ & & 109.872 & 107.734 & 117.251 & 138.400 & 108.698 & 112.166 & 64.7901 & 85.1168 \\
\hline $\begin{array}{l}\text { DL- } \\
\text { Laudanine }\end{array}$ & 127.932 & 99.8756 & 109.213 & 65.9517 & 141.136 & 113.766 & 111.725 & 80.3044 & 74.2255 \\
\hline Stigmasterol & & 54.8007 & 117.714 & 69.0176 & 132.214 & 108.142 & 121.36 & 64.5956 & \\
\hline Artemetin & & 34.7891 & 84.4057 & & 81.3879 & 85.8693 & 46.5486 & 59.9996 & \\
\hline Eseramine & 92.9136 & 83.4378 & 84.9043 & 92.3684 & 96.9445 & 86.9897 & 101.644 & & \\
\hline Sitosterol & & 109.872 & 107.734 & 117.251 & 138.400 & 108.698 & 112.166 & 64.7901 & 85.1168 \\
\hline Euphorbetin & 88.1873 & & 84.5927 & & 100.684 & & & & \\
\hline Ligand & 116.73 & 88.4699 & 81.1013 & & 89.6694 & 156.001 & 131.646 & 57.856 & \\
\hline
\end{tabular}

\section{Figures}




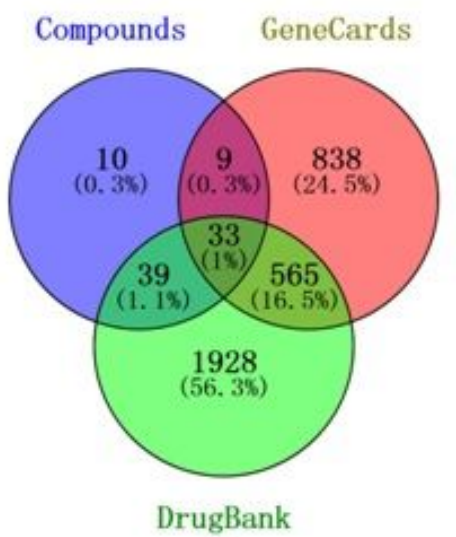

(a)

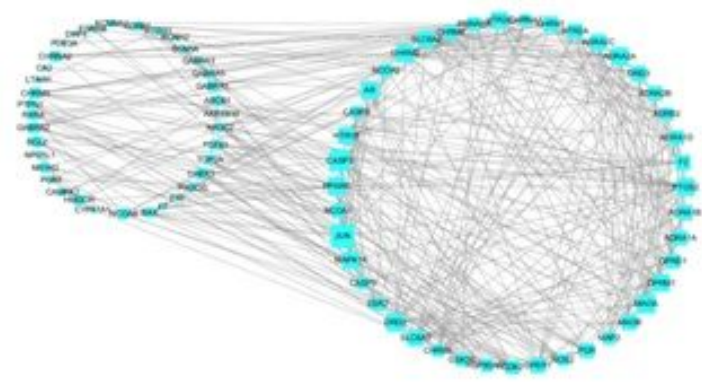

(b)

\section{Figure 1}

Targets' intersection of RL-MDR and ES network. (a) The blue circle represents targets of ES. The pink circle represents targets of reverses leukemia multidrug resistance from GeneCards. The green circle represents targets of reverses leukemia multidrug resistance from DrugBank. (b) Targets' intersection of reverses leukemia multidrug resistance and ES network: the blue circular nodes represent targets of targets intersection of reverses leukemia multidrug resistance and ES.

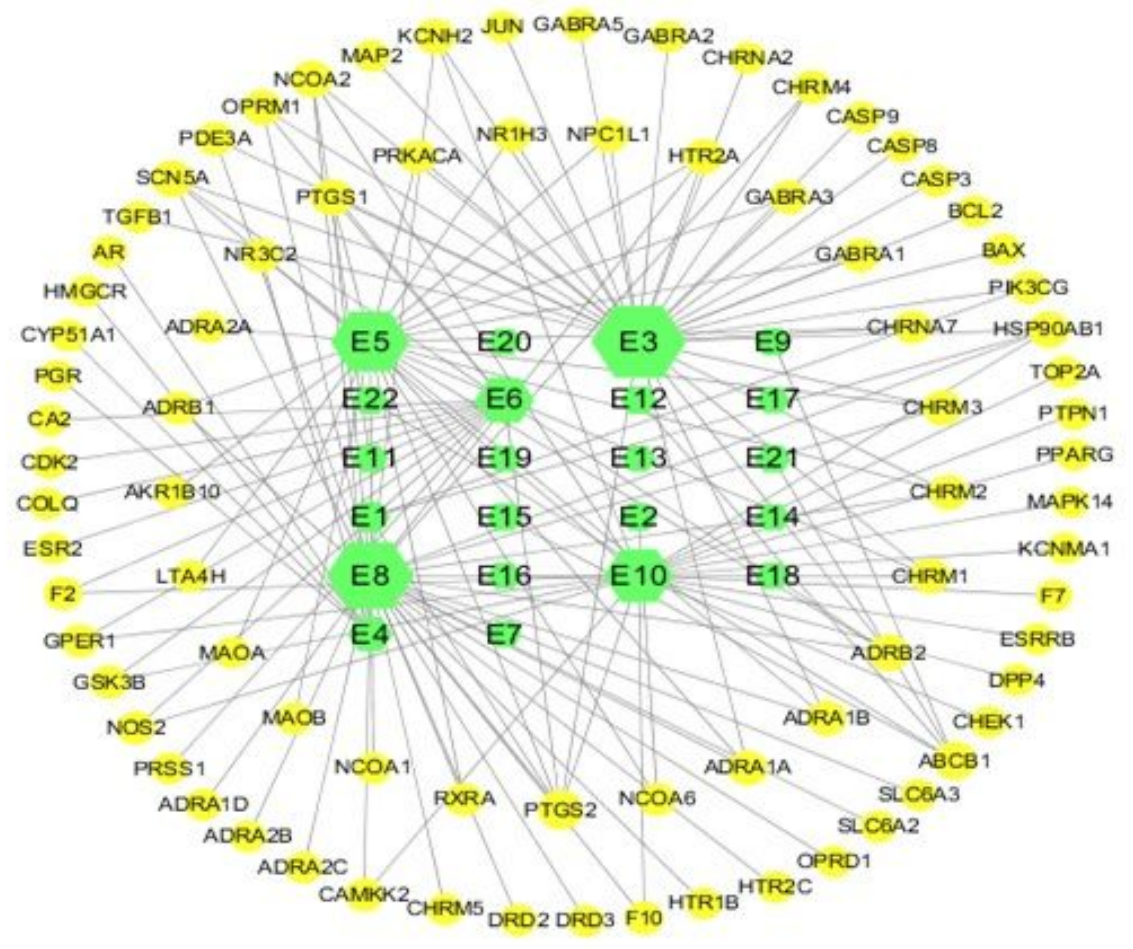

Figure 2

Compound-target network. Green hexagon nodes represent active compounds in ES. Yellow circles represent common targets between compound targets from reverses leukemia multidrug resistance and ES significant targets. Edges represent interaction between ingredients and targets. 


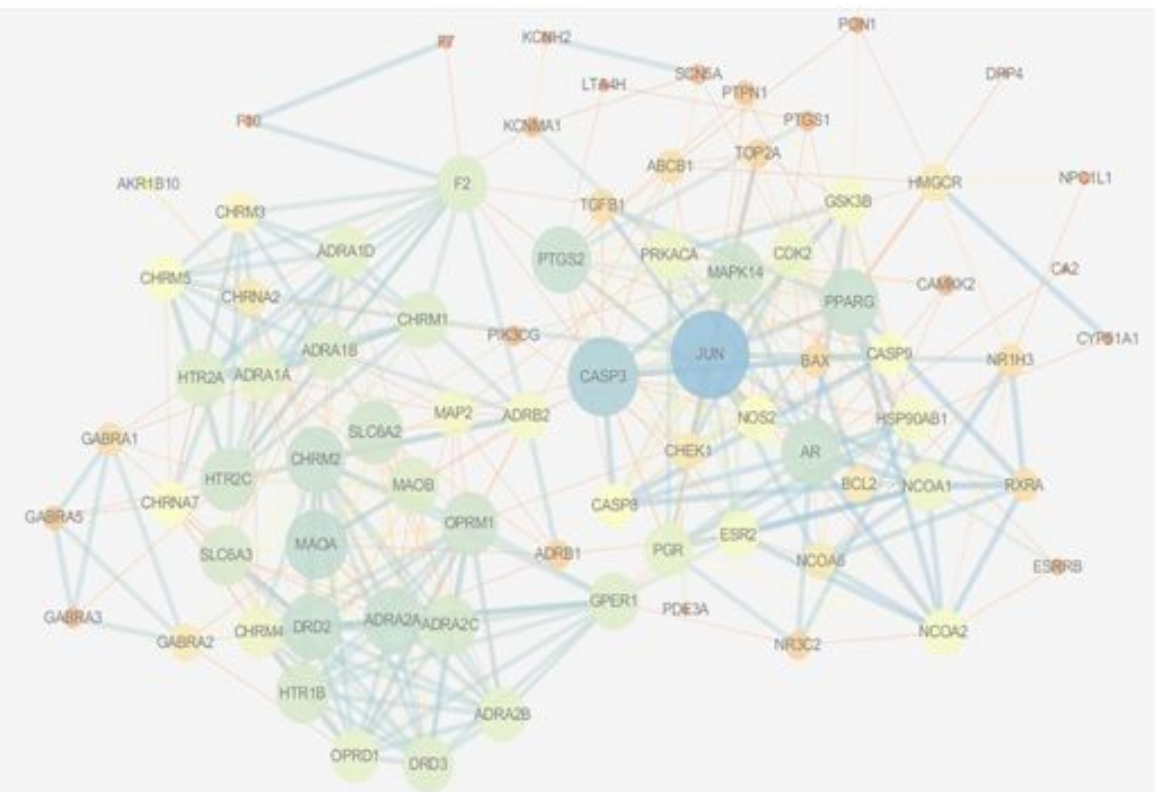

\section{Figure 3}

Protein-protein interaction network of common genes of ES-related genes of RL-MDR. Each node stands for a related target gene. The protein with greater degree is described by larger node and darker color, and the edge with greater combined score is described by thicker and darker line.
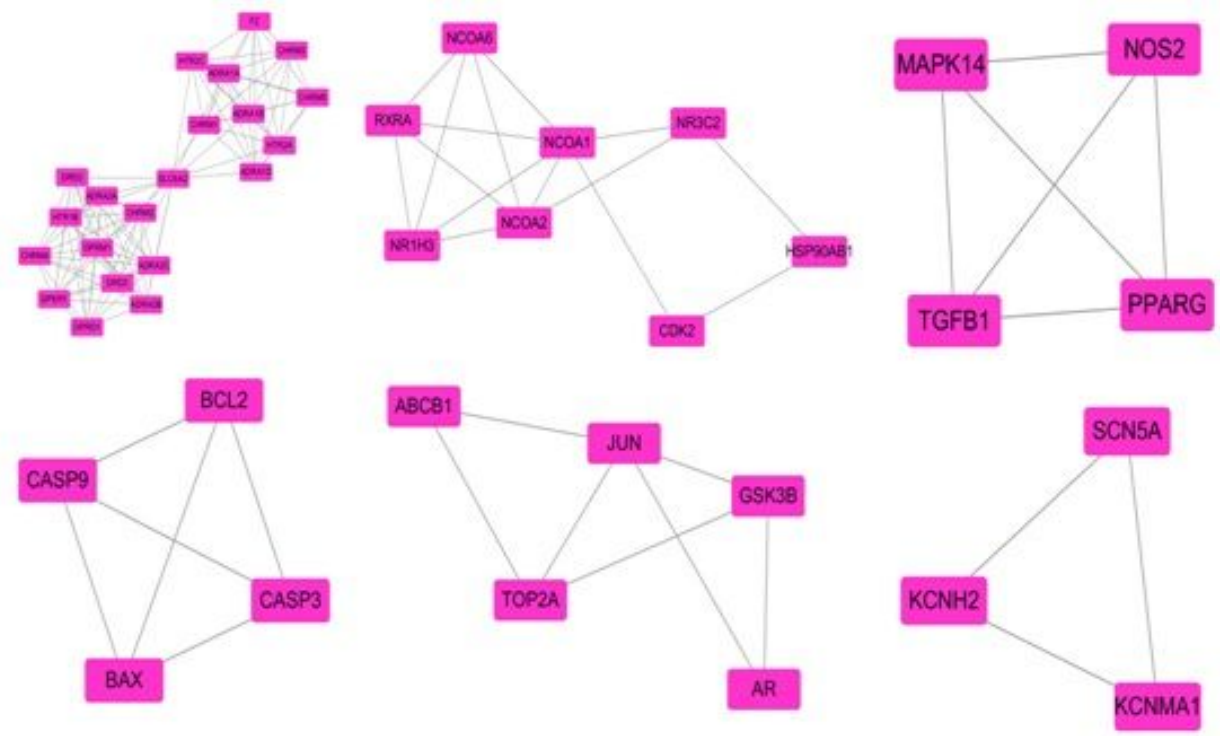

\section{Figure 4}

Interaction module of ES -RL-MDR-gene based on MCODE 


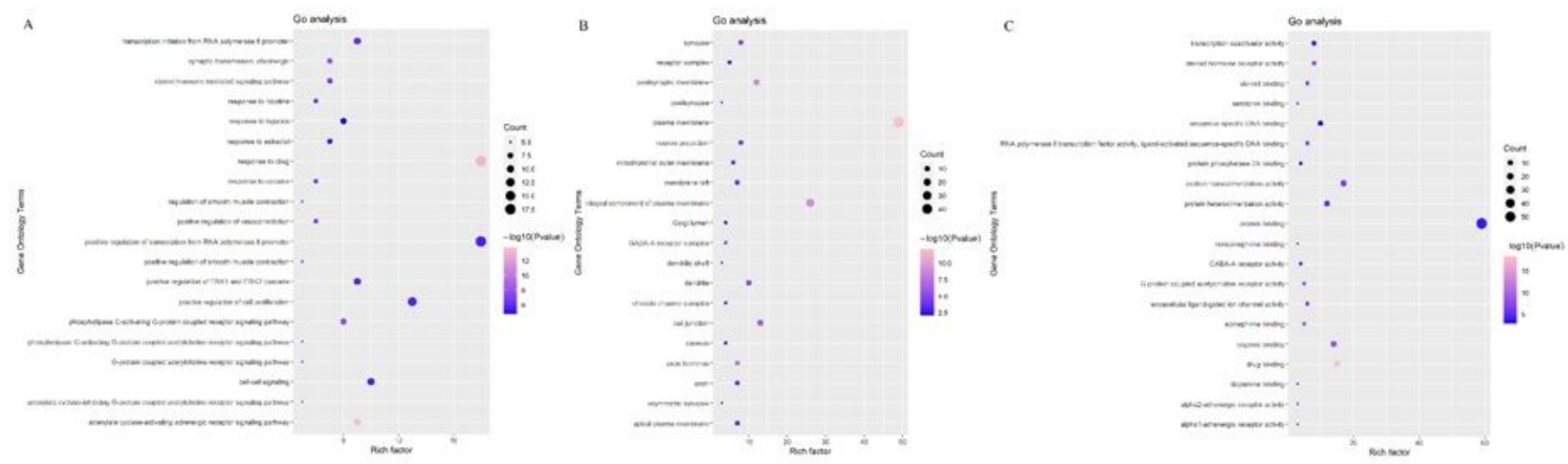

Figure 5

Gene ontology analysis of potential targets. In the bubble charts in (A-C), the Y-axis represents the names of the BP, CC, and MF terms, respectively, and the $\mathrm{X}$-axis represents the percentage-enriched $\mathrm{GO}$ categories of the target genes ( $\mathrm{P}$ value<0.01). 


\section{Top20 of pathway enrichment}

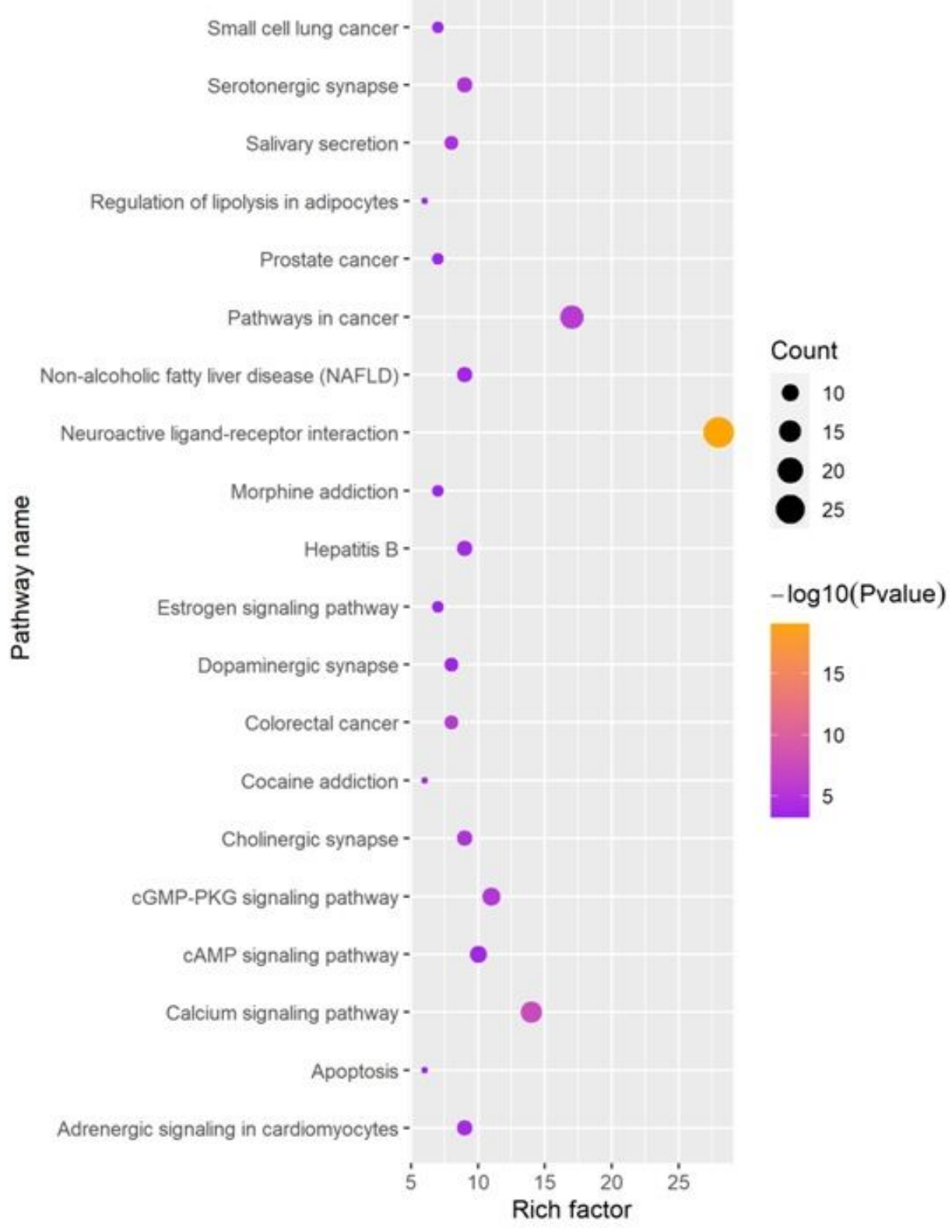

\section{Figure 6}

Enrichment analysis on KEGG pathways of key targets from active compounds of ES. Note: The size of the dots indicated the number of genes and the color of the dots reflected the -log10 (P-Value). The color of terms turned from purple to orange. The orange the bubble was, the smaller the adjusted $\mathrm{P}$ value was. 

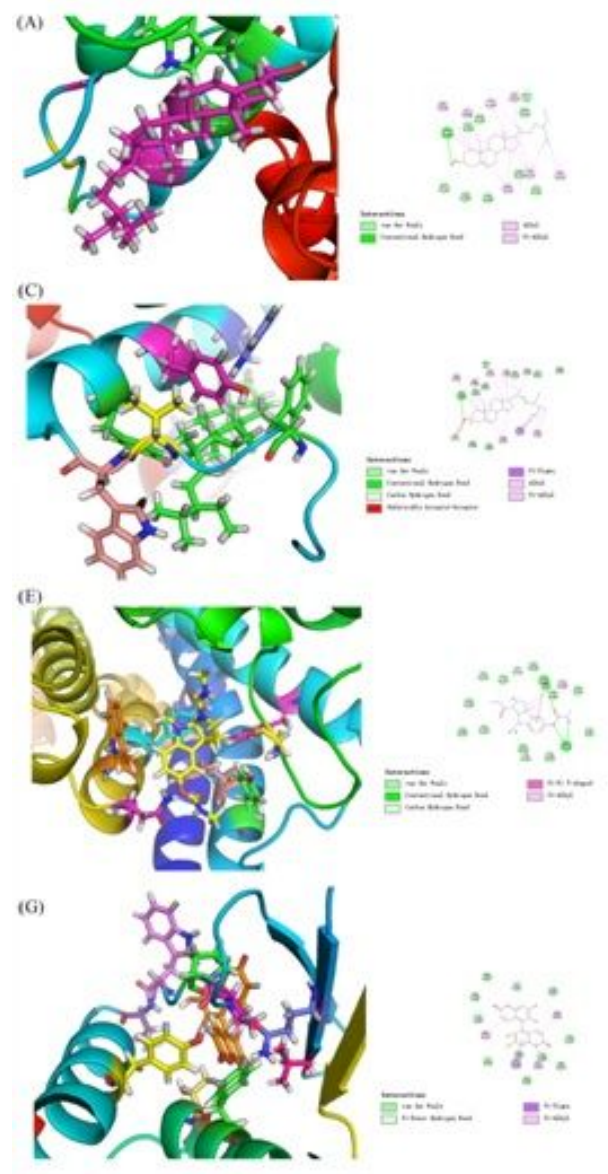

(D)
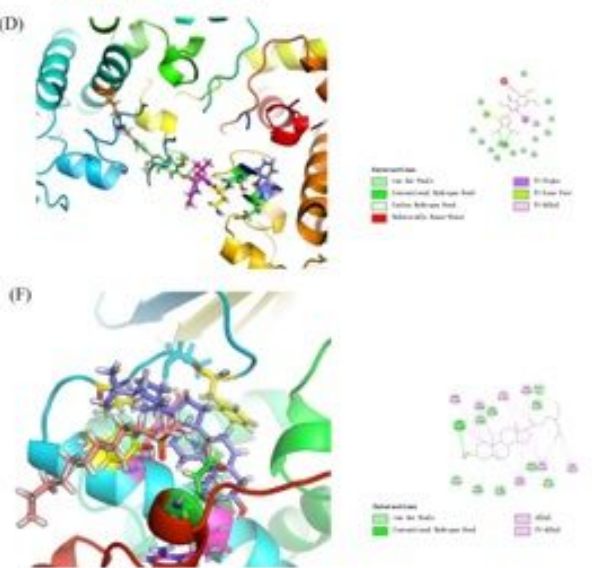

Figure 7

Molecular docking between bioactive compounds and key targets. (A): beta-sitosterol and MAOA (B): DL-Laudanine and MAOA (C): Stigmasterol and MAOA (D): Artemetin and PTGS2 (E): Eseramine and CHRM2 (F): sitosterol and MAOA (G): Euphorbetin and MAOA

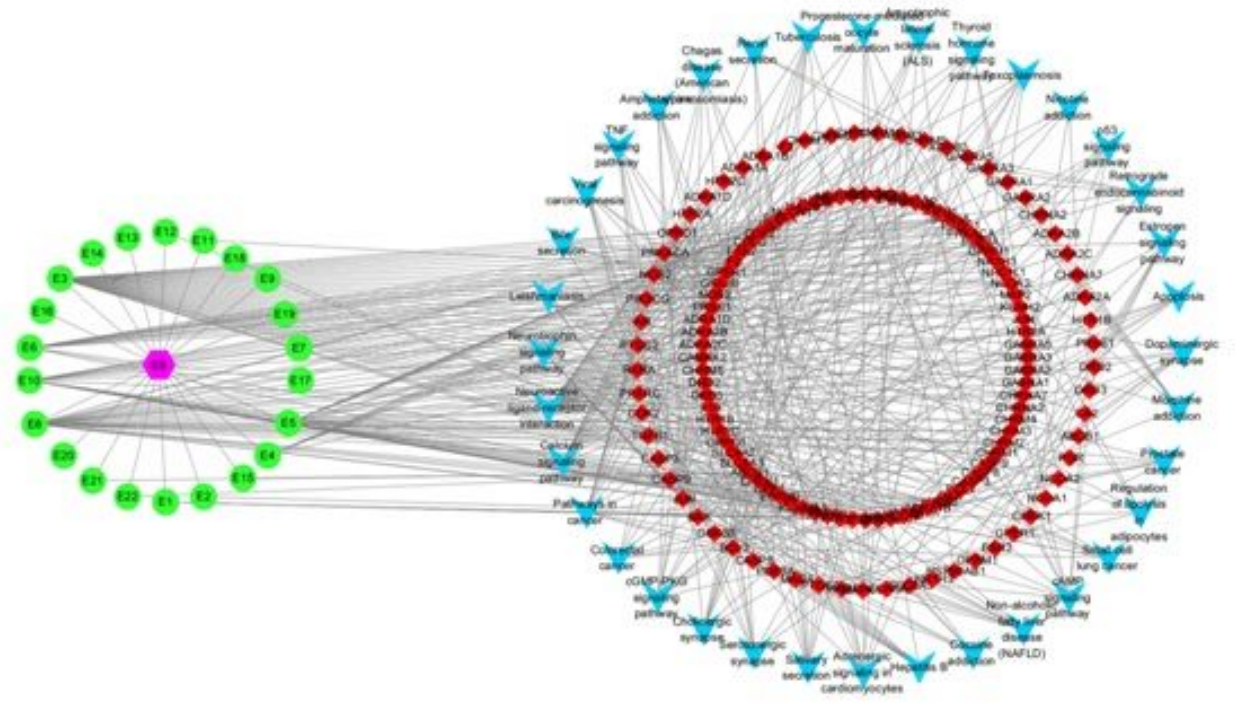

Figure 8

The herb-compound-target -pathway network. Purple hexagons represent herb. Green circles represent compounds from ES. Red diamonds represent common targets. Blue arrows represent enriched pathways. 